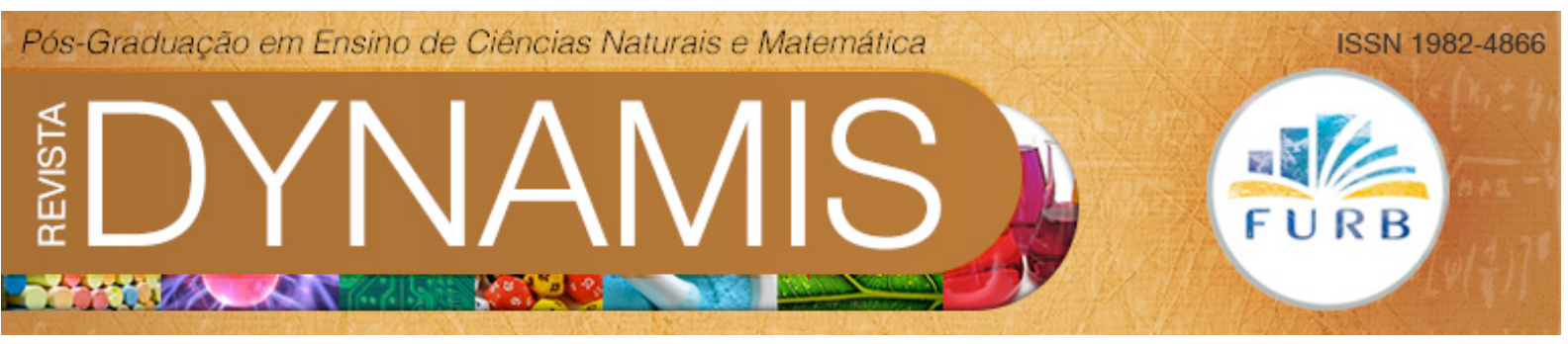

\title{
Petrofut: novos desafios para o engenheiro do futuro
}

Petrofut: new challenges to the engineer of the future

Valquíria Villas-Boas

Universidade de Caxias do Sul - UCS

vvillasboas@yahoo.com

José Arthur Martins

Universidade de Caxias do Sul - UCS

jamartin@ucs.br

Odilon Giovannini Júnior

Universidade de Caxias do Sul - UCS

ogiovanj@ucs.br

Artigo recebido em 03/12/2012. Avaliado por pares e publicado em 15/12/2012. 


\title{
Resumo
}

É possível despertar a curiosidade científica dos jovens por meio de propostas pedagógicas aplicadas fora do contexto escolar evitando, assim, a reprodução da informação das aulas tradicionais. O processo de ensino-aprendizagem através de oficinas interdisciplinares com uma estratégia inovadora pode ajudar no entendimento de conceitos científicos controversos, muitas vezes não discutidos em sala de aula e que têm importância fundamental para a formação de sujeitos críticos e conscientes. Este trabalho descreve as atividades desenvolvidas dentro do projeto UCS-PROMOPETRO: Novos Desafios para o Engenheiro do Futuro, também conhecido como PETROFUT, que promove a integração dos alunos de ensino médio com a universidade através de oficinas interativas e interdisciplinares nas áreas de Petróleo \& Gás, Biocombustíveis e Petroquímica, elaborada à luz da estratégia Aprendizagem baseada na resolução de Problemas (PBL), visando estimulá-los a seguir as carreiras científicas e tecnológicas.

Palavras chave: Interdisciplinaridade, Aprendizagem Baseada na resolução de Problemas, Ensino Médio

\begin{abstract}
One can awaken scientific curiosity in young people through educational proposals applied outside the school context, thus avoiding the reproduction of information of traditional classes. The process of teaching and learning through interdisciplinary workshops with an innovative strategy can help in the understanding of controversial scientific concepts, often not discussed in the classroom and that are of fundamental importance for the formation of critical and conscious people. This paper describes the activities carried out within the project UCSPROMOPETRO: New Challenges to the Engineer of the Future, also known as PETROFUT, that promotes the integration of high school students with the university through interactive and interdisciplinary workshops in the areas of Oil \& Gas, Petrochemicals and Biofuels, designed in the light of Problem Based Learning strategy, in order to encourage them to follow scientific and technological careers.
\end{abstract}

Keywords: Interdisciplinarity, Problem Based Learning, High School 


\section{Introdução}

As várias fases da crise do petróleo, todas ocorridas depois da Segunda Guerra Mundial, levaram vários países a desenvolver fontes alternativas de energia. No final do século passado, mais exatemente no início dos anos 70, descobriu-se que o petróleo é um recurso natural não renovável. Na época, estimou-se que em 70 anos o produto se esgotaria. Tal descoberta fez o preço do produto disparar, fazendo-o triplicar no final de 1977. Neste contexto, tão desfavorável, o Brasil foi um dos pioneiros no desenvolvimento de combustíveis renováveis, como os provenientes da madeira e da cana-de-açúcar. Por exemplo, o Pró-Álcool ou Programa Nacional do Álcool foi um programa de substituição em larga escala dos combustíveis veiculares derivados de petróleo por álcool, financiado pelo governo do Brasil a partir de 1975.

Contudo, no final de 2008, a Petrobrás anunciou o descobrimento de petróleo na camada pré-sal. A chamada camada pré-sal é uma faixa que se estende ao longo de 800 quilômetros entre os estados do Espírito Santo e Santa Catarina, abaixo do leito do mar, e engloba três bacias sedimentares (Espírito Santo, Campos e Santos). O petróleo encontrado nesta área está a profundidades que superam os 7 mil metros, abaixo de uma extensa camada de sal que, segundo geólogos, conservam a qualidade do petróleo. Estimativas apontam que a camada, no total, pode abrigar algo próximo de 100 bilhões de boe (barris de óleo equivalente) em reservas, o que colocaria o Brasil entre os dez maiores produtores do mundo (OPEC , 2012). No entanto, para que este cenário se confirme não bastam somente as reservas, será também necessário toda uma infraestrutura física e capital humano altamente qualificado. Serão necessários, principalmente, muitos engenheiros para atuarem nos diversos seguimentos da indústria de petróleo, ou seja, nas áreas de exploração, produção e transporte de petróleo e gás natural, refinarias de petróleo, unidades de processamento de gás natural, empresas de engenharia, órgãos governamentais relacionados à indústria do petróleo, comercialização, etc.

Então, aqui cabe a questão: há no Brasil um número suficiente de engenheiros para enfrentar este desafio? A resposta para esta pergunta, infelizmente, é negativa. No final de 2011, segundo dados da Confederação Nacional da Indústria (CNI), o Brasil precisaria de mais 150 mil engenheiros até o final de 2012, devido aos investimentos no setor de energia e infraestrutura, e devido à descoberta do pré-sal (PORTAL ESTADÃO, 2011). Além disso, o setor de petróleo e gás cresceu $8,7 \%$ entre 2000 e 2010, abaixo apenas do setor da indústria extrativa mineral (MACIENTE E ARAÚJO, 2011). Estava assim confirmado que o setor de petróleo e gás é um dos que têm maior falta de profissionais e exigirá ainda mais nos próximos anos, em um cenário onde o país forma uma média de 45 mil engenheiros por ano, consideradas todas as especializações de engenharia. Maciente e Araújo (2011) estimam entre 600 mil e 1,5 milhões o número de engenheiros requeridos pelo mercado de trabalho formal em 2020, dependendo da taxa de crescimento do país,

E, considerando o contexto tão propício da descoberta do pré-sal e a evidente falta de engenheiros na área de petróleo e gás, a Financiadora de Estudos e Projetos (FINEP) do Ministério da Ciência, tecnologia e Inovação lançou, no segundo semestre de 2009, uma chamada pública cujo objetivo era selecionar propostas para apoio financeiro a projetos inovadores que promovessem maior interação de instituições de nível superior com as atividades de ensino de ciências exatas e naturais nas instituições de ensino de nível médio, visando a despertar vocações e direcionar mais e melhores estudantes para as áreas tecnológicas abrangidas pelos setores de Petróleo \& Gás, Biocombustíveis e Petroquímica.

Segundo Fernandes (1998), a maioria dos estudantes considera as aulas de ciências como uma disciplina cheia de nomes, ciclos e tabelas a serem decorados. Assim, a questão que se coloca é: como atrair os estudantes ao estudo e como estimular seu interesse e 
participação? A resposta, claro, não é simples e nem há uma receita pronta. Contudo, é necessário buscar soluções, refletir sobre o assunto e trocar experiências.

Propor metodologias de ensino-aprendizagem que visam despertar o interesse do estudante para as Ciências é uma forma de promover o gosto pelas áreas científicas e tecnológicas. É fundamental que estas metodologias levem em consideração as ideias prévias dos estudantes e as habilidades que eles possuem para relacionar as entidades concretas e/ou abstratas envolvidas na relação analógica estabelecida. Atividades voltadas para a construção e reformulação de modelos pode ser uma boa opção na tentativa de atender às exigências contemporâneas para o ensino de ciências na engenharia.

É possível despertar a curiosidade científica dos jovens por meio de propostas pedagógicas inovadoras em seu contexto escolar, evitando a reprodução da informação das aulas tradicionais, que mais preparam os estudantes para as provas e que pouco os envolve no processo de conhecer e na emoção da descoberta. Através de atividades interativas e significativas é possível envolver os estudantes do ensino médio e motivá-los pelas áreas das ciências e tecnologias.

Neste contexto, o projeto UCS-PROMOPETRO: Novos Desafios para o Engenheiro do Futuro (PETROFUT), promovido pela Universidade de Caxias do Sul com apoio financeiro da FINEP, tem como finalidade principal fortalecer o ensino das ciências e despertar nos jovens o interesse pela carreira de engenheiro.

As atividades desenvolvidas neste projeto são planejadas para dar sentido e fundamentação aos ensinamentos das ciências exatas e naturais e para a aplicabilidade da teoria na solução de problemas reais, articulando aspectos científicos, econômicos, ambientais, políticos, sociais, além de reforçar o importante papel da engenharia na sociedade e nos setores industriais e de serviços. Desta forma, este projeto tem tido como objetivo integrar os estudantes de ensino médio na no processo de formação do engenheiro do futuro.

Este artigo relata, portanto, a implementação deste projeto bem como as atividades promovidas pelo mesmo. A seguir é apresentada, inicialmente, a fundamentação teórica, e depois segue com a metodologia, a estratégia pedagógica utilizada na elaboração das atividades, o desenvolvimento do projeto e, finalmente, as conclusões.

\section{Fundamentação Teórica}

A problemática do ensino-aprendizagem aparece na epistemologia bachelardiana com um enfoque próprio ao defender que aprender é uma mudança na constituição psíquica do sujeito. Isto é, aprender é superar os obstáculos que se interpõem no processo de aquisição do conhecimento. Bachelard (1996) argumenta que para que haja aprendizagem é importante que o estudante rompa com os obstáculos que impedem a compreensão dos conceitos científicos. Desta forma, o objetivo central do ensino de Ciências não deve ser aulas expositivas para a aquisição de uma grande quantidade de conteúdos, mas a superação dos obstáculos que impedem a compreensão do pensar e fazer ciência, na atualidade (CARVALHO FILHO, 2006).

A educação em Ciências deve proporcionar aos estudantes a oportunidade de desenvolver capacidades que neles despertem a inquietação diante do desconhecido, buscando explicações lógicas e razoáveis, levando os estudantes a desenvolverem posturas críticas, realizar julgamentos e tomar decisões fundamentadas em critérios objetivos, baseados em conhecimentos compartilhados por uma comunidade escolarizada (BIZZO, 1998). O significado de aprender ciência vai além de permitir o desenvolvimento cognitivo do ser humano, pois pode desenvolver também habilidades e competências procedimentais e 
atitudinais, preparando o estudante para tarefas futuras, sendo um veículo de informação que permite ao indivíduo tomar decisões fundamentadas, contribuindo assim para a formação de futuros engenheiros.

A construção de novos conhecimentos deve sempre partir do conhecimento prévio dos estudantes, mesmo que intuitivos e derivados, levando-se em consideração que o processo de aprendizagem implica a desestruturação e consequente reformulação dos conhecimentos através do diálogo e reflexão (MORAES, 1998).

Propiciar inovações no aprender e no ensinar não é apenas uma necessidade, é uma imposição do momento histórico educacional. É necessário agir de modo a melhorar o ensino atual, colaborar com a formação continuada de professores, promovendo melhorias na qualidade do ensino. Criar ambientes que propiciem aos professores e estudantes lidarem com problemas, estudo de casos, desafios, intervenções em situações reais, construindo possibilidades de argumentações e de ações conjuntas, parece ser uma alternativa de qualidade para a melhoria das relações em educação.

Fracalanza et al. (1986), propõem a substituição do verbalismo das aulas expositivas, e da grande maioria dos livros didáticos, por atividades experimentais. Segundo Lima et al. (1999), a experimentação inter-relaciona o aprendiz e os objetos de seu conhecimento, a teoria e a prática, ou seja, une a interpretação do sujeito aos fenômenos e processos naturais observados, pautados não apenas pelo conhecimento científico já estabelecido, mas pelos saberes e hipóteses levantadas pelos estudantes, diante de situações desafiadoras.

A organização das atividades em torno de problemas e hipóteses possibilitam, por um lado, superar a concepção empirista que entende que o conhecimento se origina unicamente a partir da observação e, por outro lado, relacionar o conteúdo a ser aprendido com os conhecimentos prévios dos estudantes. Entretanto, problemas dessa natureza geralmente não se enquadram bem em disciplinas específicas, exigindo uma abordagem interdisciplinar. Isto nos leva a outra característica das experimentações construtivistas que é o envolvimento de várias disciplinas ao mesmo tempo, sendo possível demonstrar para os estudantes que todas elas estão interligadas (MORAES, 1998).

As atividades práticas podem, assim, funcionar como um contraponto das aulas teóricas, como um poderoso catalisador no processo de aquisição de novos conhecimentos, pois a vivência de uma certa experiência facilita a fixação do conteúdo a ela relacionado, descartando-se a ideia de que as atividades experimentais devem servir somente para a ilustração da teoria (CAPELETTO, 1992)

Neste mesmo contexto, Pacheco (2000) afirma que os estudantes devem se confrontar com atividades de caráter investigativo e diante de um fenômeno em estudo, imprimir suas próprias concepções. É fundamental que o estudante seja instigado a propor uma explicação e confrontá-la com o conhecimento científico estabelecido, gerando um conflito cognitivo, um dos motores da evolução conceitual.

Além de ser um local de aprendizagem, o laboratório é um local de desenvolvimento do estudante como um todo, pois oportuniza que os estudantes exercitem habilidades como cooperação, concentração, organização e manipulação de equipamentos, bem como, vivenciar o método científico, entendendo como tal a observação de fenômenos, o registro sistematizado de dados, a formulação, o teste de hipótese e a inferência de conclusões.

Para Capeletto (1992) a vivência prática permite que o próprio estudante raciocine e realize as diversas etapas da investigação científica, incluindo, até onde for possível, a descoberta. Daí a importância da problematização, que é essencial para que os estudantes sejam guiados em suas observações. Quando o professor ouve os estudantes, sabe quais suas 
interpretações e como podem ser instigados a olhar de outro modo para o objeto em estudo (BRASIL, 1998).

A importância da experimentação no ensino de ciências é inquestionável no despertar dos futuros engenheiros. No Brasil, a preocupação com esse despertar tem sido possível por meio de incentivos e motivação gerados por chamadas públicas da FINEP, tais como, Ciência de todos, PROMOVE e PROMOPETRO. O projeto PETROFUT, que aqui será descrito, faz parte da chamada PROMOPETRO.

\section{A Metodologia Do Projeto}

O engenheiro é educado para projetar e construir soluções para problemas do mundo real. Originalmente, o ato de educar, em engenharia, costumava ter ligações muito estreitas com a sua prática, mas de forma gradual a educação em engenharia passou a ser mais e mais baseada na teoria (GRAAFF e CHRISTENSEN, 2004). Infelizmente, nos dias de hoje, a pedagogia dominante para a educação em engenharia ainda tem sido "o giz e o discurso", apesar de toda a pesquisa em educação que demonstra a sua ineficácia. No entanto, nos últimos anos, em muitos países esta tendência está sendo revertida.

Com o avanço da tecnologia, novos modelos de ensino-aprendizagem têm sido elaborados. Hoje em dia, são necessários ambientes de aprendizagem em que os estudantes possam ser orientados, não só sobre onde encontrar as informações, mas também, sobre como avaliá-la, analisá-la e organizá-la, tendo em vista os seus objetivos. Os novos modelos têm sem dúvida que levar em conta que o estudante para fazer uma determinada tarefa se envolve em um processo contínuo de pensamento complexo onde combina os três tipos de pensamento: básico, crítico e criativo.

Em geral, atividades pedagógicas que considerem desafios, problematizações e competições tornam o conhecimento acessível na medida em que é transformado em capacidade de agir dos envolvidos no processo. Dar a oportunidade de professores e estudantes integrarem-se de forma variada, sistêmica, interdisciplinar e contextualizada auxilia na melhoria da qualidade do ensino e da aprendizagem nas instituições educacionais, propiciando um maior envolvimento dos estudantes no processo de sua formação e de suas escolhas profissionais, e na diminuição da evasão. Com a estratégia de Aprendizagem baseada na resolução de Problemas (do inglês Problem based Learning (PBL)), descrita a seguir, é possível promover esse processo contínuo de pensamento complexo.

\section{Aprendizagem Baseada Na Resolução De Problemas}

No desenvolvimento das atividades desse projeto a estratégia pedagógica utilizada tem sido a Aprendizagem baseada na resolução de Problemas (PBL), a fim de contemplar atividades que estabeleçam conexões entre os ensinamentos básicos das ciências exatas e naturais de nível médio e aplicações práticas das áreas tecnológicas que objetivem a solução de problemas reais no âmbito das atividades industriais e de serviços da Engenharia, inclusive àqueles voltados para questões ambientais.

Quando construímos um ambiente de aprendizagem utilizando a estratégia pedagógica da Aprendizagem baseada na resolução de Problemas, a fragmentação e compartimentação das diferentes disciplinas não serão mais a regra e o problema levará à unificação na construção de conhecimentos. Para tal, é necessário que os professores, antes responsáveis cada um por sua disciplina, assumam uma postura e uma prática interdisciplinar; que estejam juntos para estudar e compreender os fenômenos em foco e como conceitos básicos das diferentes ciências envolvidas colaboram e estão integrados na busca de uma mesma solução. Uma atitude interdisciplinar também impõe a necessidade de que, em equipe, se integre 50 
objetivos, atividades, procedimentos, atitudes e planejamentos, por meio de intercâmbio, troca e diálogo (NOGUEIRA, 1994). As disciplinas interagem entre si em distintas conexões, mas sob um mesmo tema estruturante. Professores promoverão a formação dos seus estudantes a partir de tudo que ele estudou na sua vida. O ensino baseado na interdisciplinaridade e na resolução de problemas reais proporciona uma aprendizagem estruturada e rica, pois os conceitos estão organizados em torno de unidades mais globais, de estruturas conceituais e metodológicas compartilhadas por várias disciplinas, cabendo ao estudante as sínteses sobre os temas estudados.

Para o ensino de Ciências e Matemática, cujas competências fundamentam a formação em áreas tecnológicas, a contextualização e a interdisciplinaridade são pressupostos centrais para implementar um ensino por competências, como está explicitamente preconizado nos Parâmetros Curriculares Nacionais $(\mathrm{PCN})$ e nas Diretrizes Curriculares Nacionais para o Ensino Médio (DCNEM). Estes documentos foram elaborados com a finalidade de fornecer elementos que possam contribuir para "ampliar o debate nacional sobre o ensino dessa área de conhecimento, socializar informações e resultados de pesquisas, levando-as ao conjunto de professores brasileiros" (BRASIL, 2002a; BRASIL, 2002b).

A proposta deste projeto parte do principio que as representações sobre a contextualização de conteúdos das áreas de Ciências Exatas e Matemática, preconizadas nos PCN, referem-se a aspectos como a relação entre sujeito e objeto, o papel do estudante como participante e não como sujeito passivo, o ato de compreender, inventar, reconstruir; a relação com as áreas e aspectos presentes na vida social, pessoal e cultural do estudante, entre outros. Dentre esses elementos, os Parâmetros Curriculares Nacionais sugerem com maior ênfase que o ensino seja realizado a partir da proposição, em sala de aula, de conteúdos que evidenciem para os estudantes aplicações práticas, relacionadas a situações problematizadoras que estimulem os estudantes a pensar, a planejar a busca de uma solução, desenvolvendo sua autonomia intelectual.

Além de favorecer a construção de conhecimentos, a estratégia PBL propõe contribuir para o desenvolvimento de alguns atributos profissionais não técnicos considerados importantes para a prática da engenharia em uma sociedade em rápida transformação (GRAAFF E KOLMOS, 2007).

Desenvolvido na educação médica na década de 70 na reestruturação curricular do curso de medicina da Universidade de McMaster (WILKERSON, 1996), a estratégia PBL tem sido adaptada em um número crescente de áreas de atuação, incluindo a Engenharia, e em diferentes níveis educacionais. É uma estratégia pedagógico/didática centrada no estudante que pode ser utilizada para tentar desenvolver uma alfabetização científica. Sob uma ótica bem simples, PBL pode ser definida como um método instrucional que usa um problema para iniciar, focar e motivar o aprendizado.

Segundo Santos et al. (2009), as atividades são planejadas considerando-se os estudantes como protagonistas e co-responsáveis por seus aprendizados, enquanto o professor torna-se um mediador desse processo. A PBL se caracteriza como uma estratégia que utiliza um problema ou projeto como ponto de partida para aquisição de novos conhecimentos.

O problema, que representa um contexto, é caracterizado por possuir informações insuficientes, na qual o estudante possa tomar certas decisões que conduzam à sua resolução (CHARLIN, 1998). Além de conferir ao aprendizado uma finalidade motivadora, pois utiliza problemas próximos da realidade, a PBL pode favorecer a participação ativa e constante dos estudantes, bem como a interação e a troca de informações entre eles durante o processo de ensino-aprendizagem. 
Entretanto, o desenvolvimento de um processo efetivo para resolução de problemas é só um dos objetivos da PBL. Em Waters e McCracken (1997), os autores enfatizam:

Este método também pretende apoiar os estudantes na aquisição de uma base de conhecimento estruturada em torno de problemas da vida real e no desenvolvimento de competências e atitudes, incluindo trabalho em equipe e habilidades de auto-aprendizado, cooperação, ética e respeito aos pontos de vista de outras pessoas.

Como um método instrucional, PBL é consistente com os princípios da abordagem construtivista, que defende que o quê as pessoas entendem é uma função do conteúdo, contexto, atividades e objetivos do aprendiz. Peterson (1997) ressalta três importantes critérios que promovem um aprendizado mais eficaz com o uso de PBL:

a) O aprendizado acontece em um ambiente onde os estudantes estão imersos na prática e em atividades em que recebem feedback de seus colegas estudantes e professores;

b) Os estudantes recebem guias e suporte de seus pares, de maneira a promover um ensino multidirecional envolvendo outros estudantes, professores e monitores, diferentemente do ensino convencional, normalmente unidirecional (professor para estudante);

c) O aprendizado é funcional, a partir de problemas reais.

\section{Desenvolvimento Do Projeto}

O desenvolvimento do projeto consistiu em duas etapas: seleção dos candidatos e realização das oficinas.

\subsection{Seleção Dos Candidatos}

Os alunos e professores foram selecionados nas escolas de ensino médio na região de abrangência da Universidade de Caxias do Sul, considerando a participação destas escolas no projeto ENGFUT (VILLAS-BOAS, 2010), e a manifestação de interesse por parte da direção e dos professores.

A estratégia utilizada para a seleção foi a seguinte:

a) Visita à escola para detalhar o projeto junto à direção e convite aos alunos do segundo ano do ensino médio para participarem de uma apresentação do projeto.

b) Apresentação do projeto aos estudantes, incluindo a projeção de um vídeo, e aplicação de um instrumento de avaliação, composto por questões estruturadas, que procuraram avaliar o grau de comprometimento dos alunos para participar do projeto.

Com base nas respostas dos questionários foram selecionados oito professores e 128 alunos de nove escolas de ensino médio. Os alunos foram divididos em oito grupos, cada grupo acompanhado por um professor, para participarem das oficinas que estão sendo desenvolvidas pelo projeto.

\subsection{Realização Das Oficinas}

Como mencionado anteriormente, a PBL foi adotada como a estratégia pedagógica na elaboração das atividades das oficinas do projeto PETROFUT que são oferecidas aos estudantes de ensino médio. Para tal os pesquisadores da Universidade de Caxias do Sul estudaram todos os aspectos da PBL durante seis meses e elaboraram as oficinas à luz desta estratégia. Estas oficinas foram concebidas para funcionarem como um "circuito de oficinas". 
Cada oficina consiste de 4 encontros. Cada encontro tem duração de 3 horas, uma vez por semana. Uma turma de 12 a 16 estudantes de ensino médio participam de cada oficina e formam grupos de 4 estudantes. Cada grupo trabalha na resolução de tarefas e na resolução do problema de cada oficina. A cada 4 semanas, cada turma troca de oficina e inicia uma nova onde os estudantes devem resolver um outro problema.

As oficinas oferecidas neste projeto são:

Oficina 1: Produção de Biodiesel

Oficina 2: Uso de ligonocelulósicos na produção de etanol

Oficina 3: Geração de Biogás a partir de resíduos de suínos

Oficina 4: Sismologia

Oficina 5: Calor, Meio Ambiente e usos de energia

Oficina 6: Robótica

Oficina 7: Robótica no Ensino de Ciências

Oficina 8: Utilização de resíduos de Petróleo \& Biodiesel na produção de compósitos

\subsection{Avaliação Do Projeto}

Ao longo da realização das oficinas foram aplicados instrumentos para avaliarem diversos aspectos das oficinas como organização, metodologia, o ensino, a aprendizagem, etc.

As avaliações mostram que as atividades práticas e interativas são os pontos positivos das oficinas; em alguns casos, os alunos fazem uma comparação e revelam a quase inexistência desse tipo de atividade na escola. A metodologia adotada nas oficinas também é muito bem aceita. De certa forma, esses dados indicam que a escola tradicional ainda está muito aquém daquilo que os PCNs preconizam, o ensino baseado na contextualização e na interdisciplinaridade. É importante, portanto, a continuação destas atividades tanto para alunos como para os professores do ensino médio.

Contudo, ao final da fase 1 de execução das oficinas com os estudantes de ensino médio, contabilizou-se que mais de $75 \%$ dos estudantes haviam abandonado as atividades do projeto, sendo que a grande maioria (95\% dos $75 \%$ ) eram alunos de escolas públicas. A desistência destes alunos teve como maior causa a necessidade de trabalharem durante o dia e estudarem durante a noite, o que impossibilitou a continuação dos mesmos no projeto. Para a fase 2 foi necessário convidar mais estudantes, sendo que a maioria que aceitou participar eram procedentes de escolas da rede privada.

Em relação aos professores que participaram do projeto, somente um terço deles concluiu totalmente as atividades do projeto. As participações desses professores conferiram aos mesmos um certificado de participação em um curso de atualização em "Aprendizagem baseada na resolução de problemas". Observou-se, contudo, que um certificado de curso de atualização não é muito atrativo para os professores da rede pública, uma vez que não lhes confere grandes mudanças no quesito "ascensão na carreira".

\section{Considerações Finais}

De forma geral, o projeto PETROFUT tem alcançado seu objetivos. Mesmo com a evasão de alunos e professores das escolas públicas das atividades do projeto, as diversas oficinas do projeto PETROFUT têm contribuído para a divulgação dos setores de Petróleo \& Gás, Biocombustíveis e Petroquímica entre os alunos do ensino médio, contribuindo, assim, 
para que um maior número estudantes que possam vir a escolher serem engenheiros, cientistas e/ou professores das áreas de ciências e matemática. Estudos para determinar as futuras escolhas de carreiras dos alunos participantes do projeto PETROFUT estão em desenvolvimento. Finalmente, espera-se que as ações promovidas no projeto PETROFUT contribuam de forma global para uma melhor formação dos engenheiros do futuro.

\section{Referências}

BACHELARD, G. A formação do espírito científico: contribuição para uma psicanálise do conhecimento. Trad. Estela dos Santos Abreu. Rio de Janeiro: Contraponto, 1996. 316 p.

BIZZO, N. Ciências: fácil ou difícil. Ed. Ática, São Paulo, 1998. 144p.

BRASIL. Secretaria da Educação Fundamental. Parâmetros Curriculares Nacionais: Ciências Naturais. Secretaria de Educação Fundamental. Brasília: MEC/SEF, 1998.

BRASIL. Ministério da Educação. Secretaria da Educação Média e Tecnológica. Parâmetros Curriculares Nacionais: ensino médio - PCN. Brasília: MEC; SEMTEC, 2002a.

BRASIL. Ministério da Educação. Secretaria da Educação Média e Tecnológica. PCN+ Ensino Médio: orientações complementares aos Parâmetros Curriculares Nacionais. Ciências da Natureza, matemática e suas Tecnologias. Brasília: MEC; SEMTEC, 2002b.

CAPELETTO, A. Biologia e Educação ambiental: Roteiros de trabalho. Editora Ática, 1992. $224 p$.

CARVALHO FILHO, J. E. C. Educação científica na perspectiva Bachelardiana: ensino enquanto formação. Ensaio Pesquisa em Educação em Ciências, Belo Horizonte, v. 8, n. $1,1-24,2006$.

CHARLIN, B. MANN, K. HANSEN, P. The many faces of problem-based learning: a framework for understandins and comparison. Medical Teacher. V. 20, n.4, 1998.

FERNANDES, H. L. Um naturalista na sala de aula. Ciência e Ensino. Campinas, v.5, 1998.

FRACALANZA, H.; AMARAL, I. A.; GOUVEIA, M. S. F. O Ensino de Ciências no $1^{\circ}$ grau. São Paulo: Atual. 1986. p.124.

GRAAFF, E. de, CHRISTENSEN, H. P. Editorial: Theme issue on active learning in engineering education. European Journal of Engineering Education, vol. 29 (4), 2004.

GRAAFF, E.; KOLMOS, A. Management of Change Implementation of Problem-Based and Project-Based Learning in Engineering, Netherlands: Sense Publishers, 2007.

LIMA, M.E.C.C.; JÚNIOR,O.G.A.; BRAGA,S.A.M. Aprender ciências-um mundo de materiais. Belo Horizonte: Ed. UFMG. 1999. 78p.

MACIENTE, A. N.; ARAÚJO, T. C. A demanda por engenheiros e profissionais afins no mercado de trabalho formal, Radar, Brasília: Ipea, n. 12, fev. 2011. Disponível em http://www.ipea.gov.br/portal/index.php?option=com_alphacontent\&view=alphaconten t\&Itemid=80. Acesso em 01/10/2012. 
MORAES, R. O significado da experimentação numa abordagem construtivista: $O$ caso do ensino de ciências. In: BORGES, R. M. R.; MORAES, R. (Org.) Educação em Ciências nas séries iniciais. Porto Alegre: Sagra Luzzato. 1998. p. 29-45.

NOGUEIRA, A. Contribuições da interdisciplinaridade. Petrópolis: Vozes, 1994.

OPEC, Organization of the Petroleum Exporting Countries, 2012 http://www.opec.org/opec_web/en/data_graphs/330.htm. Acesso em 01/10/2012.

PACHECO, D. A Experimentação no Ensino de Ciências. Ciência e Ensino. Campinas, v. 2, 2000.

PETERSON, M. Skills to Enhance Problem-based Learning. Med Educ Online [serial online] 2, 3, 1997.

PORTAL ESTADÃO, 2011 http://www.estadao.com.br/noticias/vidae,brasil-precisa-de-mais150-mil-engenheiros-ate-2012,785879,0.htm. Acesso em 11/12/2011.

VILLAS-BOAS, V. UCS-PROMOVE: The engineer of the future, European Journal of Engineering Education, 35: 3, 289 - 297, 2010.

WATERS, R.; MCCRACKEN, M., Assessment and Evaluation in Problem-Based Learning. 1997.

WILKERSON L.; GIJSELAERS W. H. Bringing problem-based learning to higher education: theory and practice, Jossey-Bass, San Francisco. 1996. 